\title{
Are the Biomechanics of the Feet Considered Enough in their Contributory Relationship to Leg Length and Femoral Head Height Difference? Advanced Technology Provides New Information
}

\author{
Tim Maggs ${ }^{1}$, Steven Brownstein ${ }^{2 *}$ and Alexandros Siozos ${ }^{3}$ \\ ${ }^{1}$ DC, Director, Maggs Sports Chiropractic Biomechanics and Wellness Center Director, Sports Injuries and Biomechanics, USA \\ ${ }^{2}$ MD, Radiologist, Medical Director of Dynamic Medical Imaging, Medical Director of Spinal Kinetics, LLC Union, USA \\ ${ }^{3} \mathrm{MD}$, Consultant in Orthopaedic Surgery, Private Practice, Greece
}

Submission: March 23, 2021; Published: April 01, 2021

*Corresponding author: Steven Brownstein, MD, Radiologist, Medical Director of Dynamic Medical Imaging, Medical Director of Spinal Kinetics, LLC Union, USA

\begin{abstract}
Each foot is made up of 26 bones, 30 joints and more than 100 muscles, tendons and ligaments, all of which work together to provide support, balance and mobility. Our feet are responsible not only for absorbing a portion of the reaction force from the ground but for transmitting these loads to our knees, hips and back as well and, therefore, our feet function as the foundation of the whole body if we see the human body as a structure. Still, little action has been taken so far regarding studying the biomechanics of the feet (and of the whole body) and explaining why the pain and other ailments persist at times. Even if no complaints are present, a digital laser scan of the foot provides us with much critical information about balance, stability, and support of the feet and upwards. This technology allows the musculoskeletal injuries experts to better understand the lying causes of many issues and disorders and to tackle most muscle imbalances, leg length inequalities, premature onset of osteoarthritis and other common conditions. Using cutting edge technology on one hand and looking at the body much like a skyscraper, dependent upon the feet for balance, strength, and support on the other may be the key, which will unlock fewer injuries in the future.
\end{abstract}

Keywords: Biomechanics; Foot digital laser scan; Musculoskeletal injuries; Balance

\section{Introduction}

The human body is an amazing musculoskeletal structure, perfectly designed to function smoothly and properly. Although it is already well studied and examined, researchers keep sharing their astonishment about our body's tenacity and durability over time. One very good reason for this is the foundation of this structure, our feet. Each foot is made up of 26 bones, 30 joints and more than 100 muscles, tendons and ligaments, all of which work together to provide support, balance and mobility [1]. It is also established from extended series of human gait analysis that the joints of the hindfoot become loose to transfer power to the joints of the midfoot and forefoot as we walk and vice versa, depending on the momentum of the gait cycle. This is exactly why all researchers agree that the anatomy, biomechanics and kinetics of the foot is more complicated than the average healthcare specialist realizes. And its complexity may only be surpassed by its importance.
The maximum joint reaction forces were 8.72, 4.31, 2.65, and 3.41 body weight (BW) for the ankle, Chopart's, Lisfranc and metatarsophalangeal joints, respectively when measured while walking [2]. While jogging, the power on the patellofemoral joint is measured to be about 7 times BW and while deep squatting it can be as much as 20 times BW [3]. Kneeling may be bad for the knee or the patellofemoral joint, however it can also affect the hip joint as it also influences the joint reaction forces there. According to Houcke et al., the observed median HJRF in relation to body weight and hip flexion angle, respectively, was $22.3 \%$ body weight (\%BW) and $63^{\circ}$ for the simple chair, $22.5 \% \mathrm{BW}$ and $79^{\circ}$ for the car seat and $8.7 \% \mathrm{BW}$ and $50^{\circ}$ for the kneeling chair [4]. The amount of force put onto the load-bearing joints can be enormous and it makes sense that, at times, our joints become unable to withstand it over time. 
In an effort to cope with these incredible reaction forces, our body tries to adapt by using our muscles, which can absorb a great amount of these forces and by altering the tone of other soft tissues like tendons and ligaments. Doing so, these tissues become more tight or loose and become susceptible to even more biological changes. In turn and especially when it comes to the lower limbs, certain imbalances and inequalities tend to occur, which are minor at first, however, they can progress to the point where they become visible. What is interesting in the series of our studies and what we keep seeing in our everyday practices is that even with a minor discrepancy of $3-4 \mathrm{~mm}$ in femoral head height difference, a patient may be unable to overcome a common musculoskeletal injury and return to play may be prolonged or even impossible.

Our approach is based upon return to the basics of anatomy and biomechanics and that is why we focus on the foundation of the human body which is our feet. Our feet, like any other part of our body, have a great ability to adapt to external changes and factors (in this case ground reaction forces). When the way we stand, walk and run is not optimal (and it will most likely not be in any individual if properly tested and measured), then the arches of the feet begin to collapse affecting all the other joints as well up to our back and even our shoulders. We claim that we cannot correct these imbalances and inequalities unless we address the collapsed arches of the feet on one hand and that we cannot leave any collapsed arch untreated on the other.

\section{Materials and Methods}

In the first part of our study, we identified five different biomechanically flawed patterns and claimed that every human being falls into one of these five categories. In the second part, we focused on the importance of a digital x-ray scan integrated into the foot laser scan for femoral head height difference to be identified, measured, and treated. Now, in this third part, we focus on the importance of the digital laser foot scan and its metrics, trying to decode its findings and how clinically significant these findings are. Study participants were patients seeking care in our office over a 3-year period. These patient's interests ranged from wanting to learn of their biomechanical imbalances and had little to no symptoms to patients with specific musculoskeletal injuries either from degenerative changes, repetitive motion activity, trauma or a combination of these three. No one was acutely injured or presenting with antalgia or a compensatory posture. All patients presented in their normal postures.

In total, there were 400 participants in this third part of our study. All subjects were patients who presented for many different reasons. $48.75 \%$ (195) were male and $51.25 \%$ (205) were female. $54 \%$ (216) patients ranged between 20-59 years of age. The test that was performed on patients was a digital laser scan of their feet. Here is some technical information about the Kiosk scanner.

- $\quad$ About 6 feet tall

- $\quad$ About 2.5 feet wide

- $\quad$ About 3 feet deep

- $\quad$ Takes 26 key measurements

- 250 micrometers scan resolution

- Uses a Class 2M Diode Laser Product with line generating optics

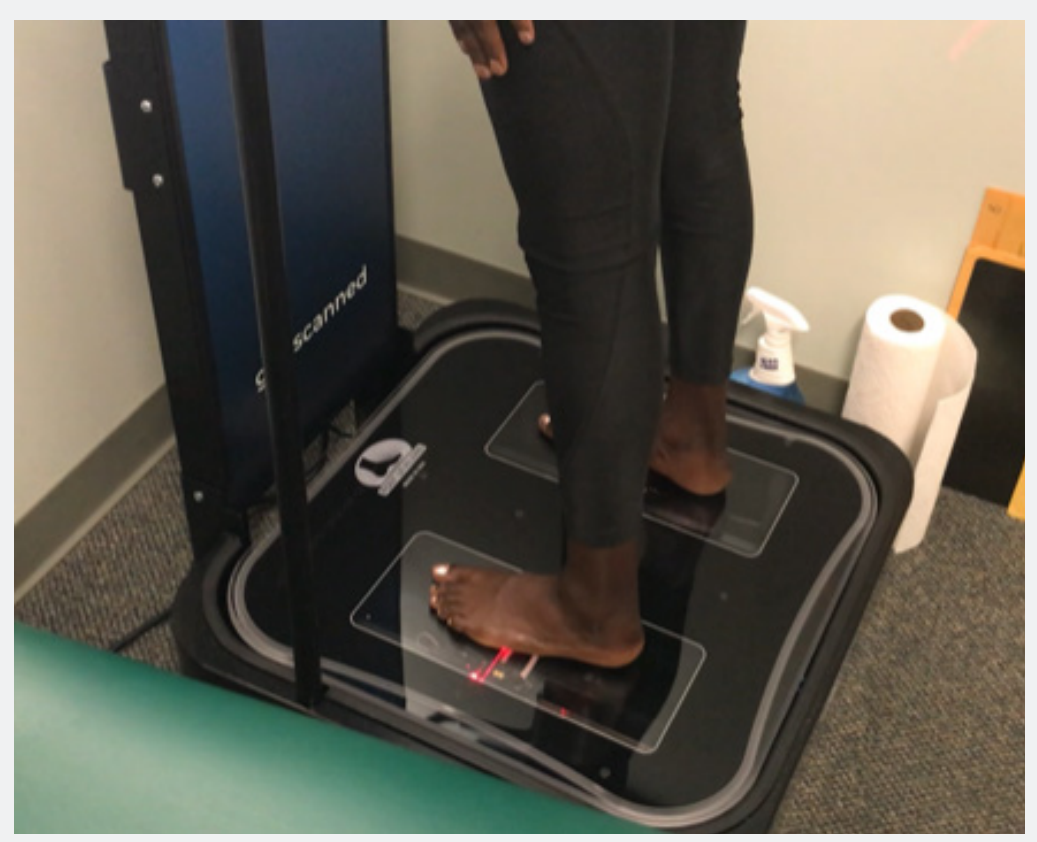

Figure 1: Digital Laser Scan of the feet. 
Our 3D scanners use a laser triangulation method to capture true 3D data. It's accurate to within 250 microns, which is one quarter of a millimeter. By capturing the foot with this much precision, we are able to determine imbalances in the plantar vault of one foot to the other. Not only does this provide detailed information for prescribing doctors to discuss with patients but it also gives our lab an accurate depiction of the patient's pedal foundation for crafting custom orthotics (Figure 1). The 3D scanner uses a moving laser to capture the entire plantar surface of the foot, developing a complete topography of the plantar vault. Colorized images are created with red pixels being closest to the laser and blue pixels being farther away. A second pass with a high-definition camera takes a scan of the foot to provide a flesh tone image as well. This allows the provider and our lab to look for sores, lesions, or other foot-related pathology.

The digital laser scan provides the following information:

Pronation Index - a number that reflects the amount of the foot that has collapsed, i.e. larger number $=$ more foot collapse. As we now know, pronation index increases with age. Since every patient in this study had some degree of collapse when scanned, these findings tell us the collapse only continues to worsen. Therefore, this issue should be addressed as early as possible to prevent the further collapse and all of the issues associated with that. Everyone needs to support their feet with orthotics.

Body Weight Distribution - 50/50 is optimal. Technically, this is pixel percentage difference. The part of the foot that contacts the digital laser scanner can be determined by pixel representation. The percentages that are seen on the report are the percentage of pixel formation on each foot, but this can easily be translated as body weight difference, as body weight governs the number of pixels on each foot. The larger ratio obviously will predispose a person to unilateral and asymmetrical loading and premature breakdown.

Arch Height Difference - a volume of measurement that indicates the difference between the collective collapse of the arches. A greater collapse is largely due to greater weight bearing on that side (Figure 2).

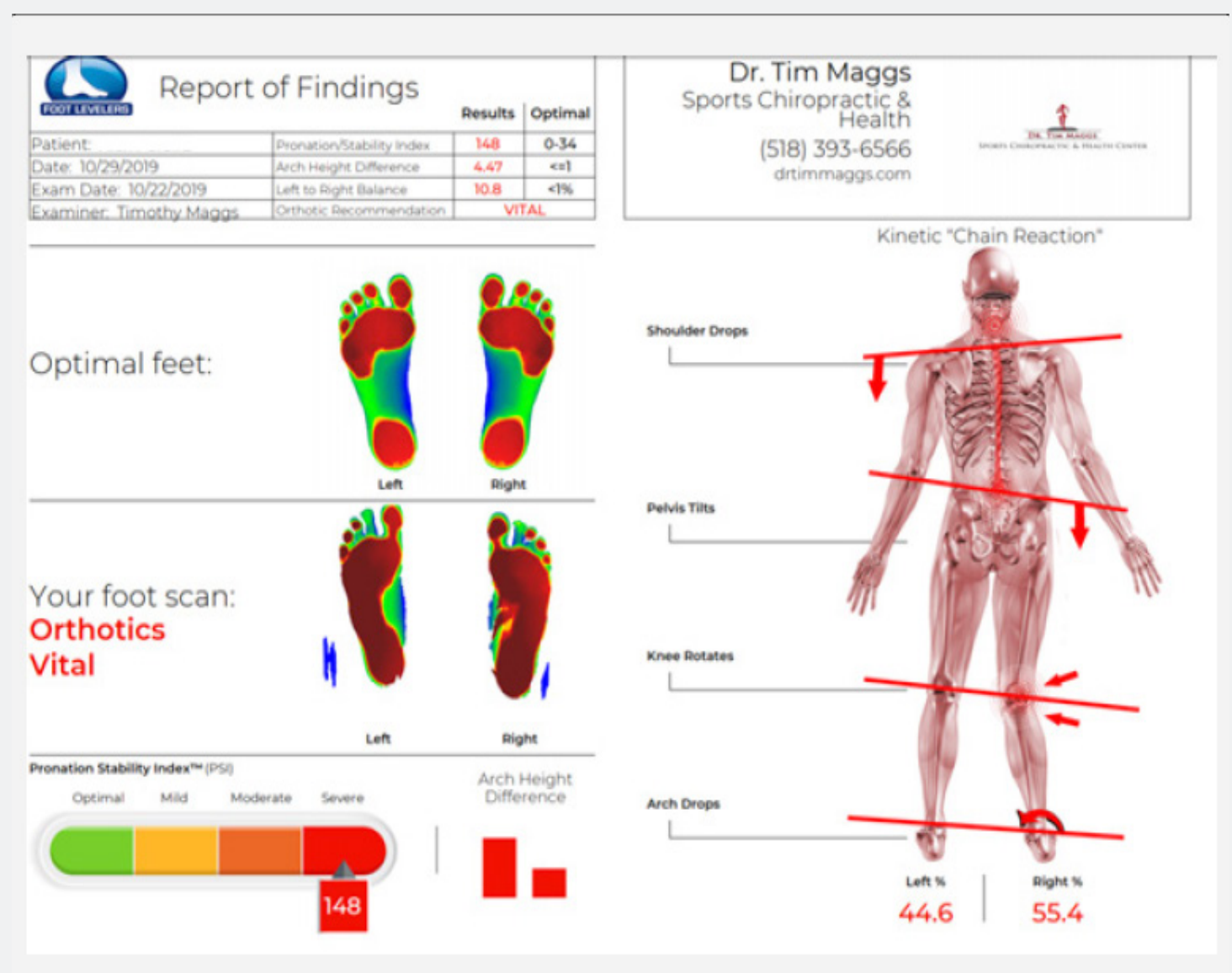

Figure 2: Laser foot scan - Report. 


\section{Orthopedics and Rheumatology Open Access Journal (OROAJ)}

\section{Results}

In this third part of our study, there were 400 participants who underwent a digital laser scan of their feet. They were instructed to hop on the platform and just stand in a neutral position/normal posture being barefoot. Each scan only took a few minutes to be completed.

As for the Pronation Stability Index (PSI), our results really highlighted how much collapse there is in the arches of the feet of the individuals out there. Optimal range of PSI is 0-34, however, 284 of them $(71 \%)$ presented with a PSI greater than 85 , which accounts for severe collapse of the foot arches and this result was consistent in all age subgroups! Women did slightly better than men overall, but we believe that no difference would be noted within a sample of thousands. The mean average of PSI in all age groups surpassed 100 with the exception of the $0-19$ group (89.77). Perhaps the most important result about the Pronation Index Number was that the older the patient was, the worse he/ she did on that specific measurement. In other words, not only almost every individual was measured to have moderate or severe collapse of his/her foot arches, but this collapse seems to only getting worse as time goes by.

We also received results with regard to body weight distribution and a comparison was made between the left and the right side. Optimal weight distribution is of course 50/50 and an optimal result would be a difference less than $1 \%$. However, when we summed the deviation ratio of the weight distribution (regardless of which side, left or right, sustained more load), we found that there was approximately a $3.12 \%$ difference on average between the two sides. This finding far exceeds the $1 \%$ mark indicating further collapse of the foot arches, which leads to the body's effort to compensate to increased loads. Our results ranged from values like $0.2 \%$ and $0.45 \%$ to more increased values like $6 \%$ or $9 \%$, with some patients' reports giving us up to $16 \%$ difference in their weight distribution! Again, no difference was mentioned between age groups and male or female patients.

As for the arch height difference, this is another way to assess the collapse of the foot arches and compare it to the contralateral side. Our scanner considers values up to 1 to be normal and in our sample the mean average was 2.39. The results for men and women were 2.6 and 2.16, respectively. No age group had a mean average less than 2 , with the 40-59 age group marking the worst performance (2.59). This was another finding, which triggers the kinetic chain reaction from the feet upwards and contributes to the formation of the Crooked Man biomechanically flawed patterns, as analysed in the previous parts of this study.

\section{Discussion}

The concern and interest of the healthcare community about the architectural structure of the human musculoskeletal system is not something new. This is exactly the reason why our arsenal has been enhanced over the years, providing us with not only solid understanding of the very principles of our body but with cutting edge technology to make use of this knowledge as well. Still, the approach of most of the musculoskeletal experts out there remains reactive. All injuries tend to be looked at because of a sudden move, a fall, a sprain or simply due to overuse. This may be true at times, however, we believe that the proactive approach is preferable because it can lead to these injuries never happening in the first place, in other words, to fewer injuries. After all, prevention is better than cure.

These are exactly the reasons that explain the core of our series of studies. To fully grasp why injuries and degeneration occur (except for accidents) or why the pain sometimes will not go away and in a fast-changing world where innovation speed exceeds our perception capacity, we decided to go back to the basics. And the basics of how we can stand, walk and run are our feet. Our feet are the first anatomical structure which bears and withstands the ground reaction forces and, therefore, serve as the foundation and the ground floor of the whole building, if we look at the human body as a living, running machine. We then chose to put our core foundation to the test and under the spotlight.

To do so, we used our long and substantial experience in everyday practice, current literature and advanced technology available. We formed a random sample of 400 participants, out of which not everyone was a "patient" but rather someone who wanted to know more about his/her biomechanical status. Each participant underwent a safe and quick digital laser scan of the feet and the information gathered showed us how these individuals are standing in their natural/normal postures in terms of weight/load distribution and foot arches' strength and stability. Regardless of what the complaint is of the patient, or even if there is no complaint, we performed this digital laser foot scan as the understanding of their architectural structure, biomechanics or musculoskeletal system can only be achieved with this information, starting as early as the age of 12 .

To be honest, we expected the results to show a mild to moderate collapse of the arches of the foot in some individuals. What struck us is the fact that $80 \%+$ of the 400 participants presented with severe Pronation Stability Index and significant uneven distribution of their body weight. Things were not looking good with regard to arch height difference either, with very few patients achieving a score of less than 1 . The deviation from the optimal 50-50 in terms of body weight distribution (left-right) was measured to be on average $3.12 \%$, again significantly higher than the optimal $1 \%$. Some of the participants recorded up to $17 \%$ difference in load distribution between the left and the right side, indicating a very hazardous predisposition to key joints being injured and become prematurely degenerated. The mean average for PSI not only exceeds 100, but keeps on increasing as we examine older patients, implying that a collapse in one arch of the foot will only get worse as time goes by. Overall, this third part 
of our study tried to illustrate the outline of the biomechanical status of the standing posture of random individuals. Our results identified way more cases of abnormal and suboptimal standing than we expected. This finding prompts us to claim that only very few present with the strength and stability that is required for an optimal weight distribution starting from the feet upwards. Taking a step even further, we suggest that these unstable and weak feet are the main reason for joint rotation, muscle and ligament imbalances and, ultimately, leg length inequalities. In turn, these changes serve as a "fertile ground" for overuse injuries and joint degeneration occurring more regularly and at a more severe level than they should.

\section{Conclusion}

The reactive approach of musculoskeletal injuries and degeneration has already cost us a lot along with controversial results. Our team would introduce the proactive approach instead, which focuses on preventing these disorders from happening in the first place. Implementing a digital laser scan of the foot of every individual from the age of 12 years old may prove to be a powerful tool towards that goal.

\section{References}

1. Anatomy of the foot (2021).

2. Yongcheol Kim, Kyoung Min Lee, Seungbum Koo (2018) Joint moments and contact forces in the foot during walking. 74: 79-85.

3. Evan Watts (2021) Patellofemoral joint.

4. J Van Houcke, A Schouten, G Steenackers, D Vandermeulen, C Pattyn, et al. (2017) Computer-based estimation of the hip joint reaction force and hip flexion angle in three different sitting configurations. Appl Ergon 63: 99-105.

\section{Your next submission with Juniper Publishers will reach you the below assets}

- Quality Editorial service

- Swift Peer Review

- Reprints availability

- E-prints Service

- Manuscript Podcast for convenient understanding

- Global attainment for your research

- Manuscript accessibility in different formats ( Pdf, E-pub, Full Text, Audio)

- Unceasing customer service

Track the below URL for one-step submission https://juniperpublishers.com/online-submission.php 